The AstrophysiCAL JournaL, 520:627-633, 1999 August 1

(C) 1999. The American Astronomical Society. All rights reserved. Printed in U.S.A.

\title{
OBSERVATIONS OF OJ 287 FROM THE GEODETIC-VLBI ARCHIVE OF THE WASHINGTON CORRELATOR
}

\author{
C. E. TATEyama \\ Centro de Rádio-Astronomia e Aplicações Espaciais/CRAAE-INPE, Instituto Presbiteriano Mackenzie, Rua da Consolação 896, \\ 01302-000, São Paulo, SP, Brazil \\ K. A. KInGHAM \\ US Naval Observatory, Earth Orientation Department, 3450 Massachusetts Avenue, Washington, DC 20392 \\ P. KAUFMANN \\ Centro de Rádio-Astronomia e Aplicações Espaciais/CRAAE-Mackenzie, Instituto Presbiteriano Mackenzie, Rua da Consolação 896, \\ 01302-000, São Paulo, SP, Brazil \\ B. G. PINER \\ Jet Propulsion Laboratory, California Institute of Technology, MS 238-332, 4800 Oak Grove Drive, Pasadena, CA 91109 \\ L. C. L. BotTI \\ Centro de Rádio-Astronomia e Aplicações Espaciais/CRAAE-INPE, Instituto Presbiteriano Mackenzie, Rua da Consolação 896, \\ 01302-000, São Paulo, SP, Brazil \\ AND \\ A. M. P. DE LUCENA \\ Rádio Observatório Espacial do Nordeste (ROEN), CRAAE-INPE, Estrada do Fio, 6000, Eusébio (Fortaleza), CE, Brazil \\ Received 1998 October 1; accepted 1999 March 8
}

\begin{abstract}
We present 27 geodetic VLBI maps of OJ 287 obtained from the archive of the Washington correlator. The observations presented here were made between 1990 October and 1996 December. During this period a sequence of six superluminal components has been identified. We measured the proper motion of these components to be approximately 0.5 mas $\mathrm{yr}^{-1}$, which is about twice as high as that seen in previous VLBI observations. These results imply a higher component ejection rate than previously observed, in good agreement with the observed occurrences of radio outbursts. We have examined a possible connection between VLBI components and optical flares in the framework of a binary black hole system.
\end{abstract}

Subject headings: BL Lacertae objects: individual (OJ 287) — galaxies: jets — radio continuum: galaxies

\section{INTRODUCTION}

OJ 287 is a highly variable radio source at $z=0.306$ (Miller, French, \& Hawley 1978; Sitko \& Junkkarinen 1985). The nature of the underlying galaxy is not well known; Kinman (1975) and Hutchings et al. (1984) detected a nebulosity that might be a galaxy. The light curve of OJ 287 presents a complex structure, but its most interesting aspect is the series of prominent flares which recur with a period of 12 yr (Sillanpää et al. 1988). Recent $V$-band observations have revealed double-peaked outbursts (Sillanpää et al. 1996; Lehto \& Valtonen 1996). To reproduce the cyclic 12 yr optical flares, OJ 287 has become the best candidate to harbor a supermassive binary black hole system. Sillanpää et al. (1988) used such a system, in which the light variations were related to tidally induced mass flows from the accretion disk into the black hole, to explain the periodicity in OJ 287's light curve. The orbit of the secondary black hole was assumed to be coplanar with the accretion disk. Lehto \& Valtonen (1996) suggested a binary-system model in which the primary is surrounded by an accretion disk with a high inclination angle relative to the orbit of the secondary. A major flare would be observed whenever the secondary crossed the disk of the primary. The binarysystem model of Villata et al. (1998), with two independent relativistic jets, and the nodding-disk model of Katz (1997) use a sweeping-beam approach to explain the same optical flares.

The radio light curves are dominated by outbursts at irregular intervals of about a year, and modulated by a much longer nonperiodic timescale (Usher 1979). Analysis of radio-optical events in OJ 287 has shown correlated activity. Usher (1979) compared events from 1966 to 1978 and proposed that most optical outbursts are synchronous with radio counterparts. Valtaoja, Sillanpää, \& Valtaoja (1987) studied isolated flaring events and found that optical variations preceded the radio ones by a few months.

VLBI observations of OJ 287 have been made at $5 \mathrm{GHz}$ (Roberts, Gabuzda, \& Wardle 1987; Gabuzda, Wardle, \& Roberts 1989), 8 GHz (Vicente, Charlot, \& Sol 1996), and 43 and $100 \mathrm{GHz}$ (Tateyama et al. 1996). Superluminal motion was first detected from VLBI polarization data at $5 \mathrm{GHz}$ (Roberts et al. 1987). Previous to this paper, three superluminal knots (named K1, K2, and K3) were followed from 1981 to 1988 . The observed proper motion of these components corresponds to an apparent superluminal speed of between $3.7 c$ and $5.1 c\left(H_{0}=65 \mathrm{~km} \mathrm{~s}^{-1} \mathrm{Mpc}^{-1}, q_{0}=0.5\right)$. In a single-epoch VLBI observation at $8.3 \mathrm{GHz}$, a new jet component, $\mathrm{K} 4$, and another polarization component located between the core and K4, named K5, were added to the list of components of OJ 287 by Gabuzda \& Cawthorne (1996). More recently, Vicente et al. (1996) fitted a helical path with two consecutive loops to the trajectory of component K3.

We present 27 geodetic VLBI maps at $8.3 \mathrm{GHz}$ obtained from the Washington correlator's database. Geodetic VLBI maps have been successfully used for astrophysical studies of compact sources (e.g., Guoquiang, Rönnäng, \& Bååth 1987; Charlot 1990; Britzen et al. 1994; Vicente et al. 1996). 
More recently, Piner \& Kingham (1998) and Tateyama et al. (1998) have obtained interesting results for a group of EGRET blazars and BL Lac, respectively, using the Washington VLBI correlator's database.

\section{OBSERVATIONS}

The VLBI observations used in this paper are from geodetic VLBI data archived at the Washington correlator. These data were obtained from geodetic dual-frequency VLBI experiments (Rogers et al. 1983) carried out by the Naval Observatory (Eubanks et al. 1991), the National Oceanographic and Atmospheric Administration (Carter, Robertson, \& MacKay 1985), the Crustal Dynamics Project, and the Space Geodesy Project (Coates et al. 1985; Smith \& Turcotte 1993). The VLBI observations were processed at the Washington VLBI correlator at the US Naval Observatory (USNO).

All observations were obtained with Mark III dualfrequency VLBI receivers at both the $\mathrm{X}$ and the $\mathrm{S}$ band (centered at 8.5 and $2.3 \mathrm{GHz}$, respectively), providing noise temperatures of 70-200 K. The $X$ band consists of 8 individual channels of $2 \mathrm{MHz}$ bandwidth spanning the range $8.2-8.9 \mathrm{GHz}$. All stations were equipped with $\mathrm{H}$ masers as the local frequency standards. Geodetic VLBI data useful for imaging OJ 287 begins to appear in the Washington correlator archive around 1990. Table 1 lists all observations used in this work: column (1) gives the epochs of the observations, column (2) the names of the experiments, column (3) the antennas participating in the experiments, column (4) the peak brightness of the images, column (5) the interferometric dirty beams, column (6) the position angles of the beams measured from north to east, and column (7) the number of scan baselines in the observations, where the number of scan baselines is the sum over all the scans of the number of baselines per scan. The dirty beams were about 0.5-0.6 mas in size for all maps. We have used a restored circular beam of 0.6 mas on the maps. The dynamic range, defined as the ratio of the peak flux per beam to the lowest positive contour in the maps, is about $300: 1$. The total length in which the moving components in OJ 287 can be followed with the geodetic network appears to be limited to radii not much greater than 1.5 mas. This is about half the distance reached by the knots in the $5 \mathrm{GHz}$ VLBI observations made by Roberts et al. (1987) and Gabuzda et al. (1989). Except for CRD96GH (Table 1), where the resulting map is the sum of the experiments CRD96G and CRD96H observed on 1996 September 19 and 20, respectively, all maps were obtained from a single geodetic observation.

The data were coherently averaged to $4 \mathrm{~s}$ to determine the visibilities. The data were calibrated and fringed using

TABLE 1

8.4 GHz VLBI OBSERVATIONS OF OJ 287

\begin{tabular}{|c|c|c|c|c|c|c|}
\hline $\begin{array}{l}\text { Epoch } \\
\text { (1) }\end{array}$ & $\begin{array}{l}\text { Name } \\
(2)\end{array}$ & $\begin{array}{l}\text { Antennas }{ }^{a} \\
\text { (3) }\end{array}$ & $\begin{array}{c}\text { Peak } \\
\text { Brightness } \\
\left(\mathrm{Jy} \mathrm{beam}^{-1}\right) \\
\text { (4) }\end{array}$ & $\begin{array}{c}\text { Synthesized } \\
\text { Beam } \\
(\mathrm{mas} \times \text { mas) } \\
(5)\end{array}$ & $\begin{array}{c}\text { Beam } \\
\text { Angle } \\
\text { (deg) } \\
(6)\end{array}$ & $\begin{array}{c}\text { Number } \\
\text { of Scan } \\
\text { Baselines } \\
\text { (7) }\end{array}$ \\
\hline 1990 Oct $27 \ldots \ldots$ & NAPSA & JYEAC & 4.9 & $1.6 \times 1.3$ & 39 & 139 \\
\hline 1992 Aug $03 \ldots \ldots$ & IR752 & GJREV & 2.3 & $1.8 \times 0.7$ & -19 & 48 \\
\hline 1992 Aug $10 \ldots \ldots$ & IR753 & GJREV & 2.4 & $1.0 \times 0.7$ & -15 & 53 \\
\hline 1993 Jun $24 \ldots \ldots$ & NB02 & AIWVGT & 1.3 & $1.6 \times 0.6$ & -2 & 66 \\
\hline 1993 Dec $10 \ldots \ldots$ & NB08 & GIWTV & 1.5 & $2.5 \times 0.5$ & -10 & 63 \\
\hline 1994 Sep $02 \ldots \ldots$ & NB16 & GXAIWTV & 1.0 & $1.5 \times 0.6$ & 2 & 59 \\
\hline 1994 Dec $13 \ldots \ldots$ & NE85 & FAKWVN & 1.0 & $0.5 \times 0.5$ & -35 & 63 \\
\hline 1995 Jan $25 \ldots \ldots$. & NA91 & FAKWV & 0.9 & $0.7 \times 0.4$ & 20 & 22 \\
\hline 1995 Apr $18 \ldots .$. & NA103 & FAKWVO & 0.8 & $0.9 \times 0.6$ & -21 & 51 \\
\hline 1995 Jun $14 \ldots \ldots$ & NXS6 & GAMWOV & 0.8 & $1.1 \times 0.7$ & -29 & 51 \\
\hline 1995 Sep $19 \ldots \ldots$ & NA125 & GFAKWNV & 1.2 & $1.3 \times 0.6$ & -4 & 29 \\
\hline 1995 Oct $17 \ldots \ldots$ & NA129 & GFAKHLNV & 1.4 & $0.7 \times 0.5$ & 17 & 80 \\
\hline 1995 Dec $12 \ldots \ldots$ & NA137 & FAKNOV & 2.0 & $0.7 \times 0.6$ & -75 & 63 \\
\hline 1996 Mar $05 \ldots . .$. & NA149 & FAKNOV & 1.2 & $0.8 \times 0.6$ & -39 & 62 \\
\hline $1996 \operatorname{Mar} 26 \ldots \ldots$ & NA152 & FAKNOV & 1.1 & $0.8 \times 0.6$ & -34 & 61 \\
\hline 1996 Apr $02 \ldots \ldots$ & NA153 & FAKMNV & 1.2 & $0.9 \times 0.6$ & 2 & 67 \\
\hline 1996 Apr $24 \ldots \ldots$ & NXS10 & GAKNOV & 0.9 & $0.9 \times 0.7$ & -14 & 22 \\
\hline 1996 Aug $20 \ldots \ldots$ & NA173 & FAKWNOV & 1.1 & $0.6 \times 0.5$ & 15 & 63 \\
\hline 1996 Sep $03 \ldots \ldots$ & NA175 & FAKNOV & 1.1 & $0.8 \times 0.5$ & -58 & 51 \\
\hline 1996 Sep $17 \ldots \ldots$ & NA177 & FAKNOV & 1.1 & $0.7 \times 0.6$ & -27 & 69 \\
\hline 1996 Sep $19 \ldots \ldots$ & CRD96GH & AKNOV & 0.9 & $0.8 \times 0.6$ & -22 & 204 \\
\hline 1996 Oct $01 \ldots \ldots$ & NA179 & FAKNOV & 1.2 & $1.0 \times 0.5$ & -16 & 68 \\
\hline 1996 Oct $02 \ldots \ldots$ & CRD96J & AKNOV & 1.0 & $0.9 \times 0.5$ & -18 & 98 \\
\hline 1996 Oct $03 \ldots \ldots$ & CRD96K & AKNOV & 0.9 & $1.0 \times 0.5$ & -20 & 79 \\
\hline 1996 Oct $04 \ldots \ldots$ & CRD96L & AKNOV & 1.0 & $1.0 \times 0.6$ & -23 & 91 \\
\hline 1996 Nov $12 \ldots \ldots$ & NA185 & FAKNOV & 1.3 & $0.6 \times 0.4$ & 8 & 61 \\
\hline 1996 Dec $10 \ldots \ldots$ & NA189 & FAKNOV & 1.1 & $0.9 \times 0.5$ & -46 & 62 \\
\hline
\end{tabular}

${ }^{a}$ A: Gilcreek (Alaska, 26 m); C: Hat Creek (California, 26 m); E: Westford (Massachusetts, $18 \mathrm{~m}$ ); F: Fortaleza (Brazil, $14 \mathrm{~m}$ ); G: Algopark (Ontario, $46 \mathrm{~m}$ ); H: MK-VLBA (Hawaii, $25 \mathrm{~m}$ ); I: Matera (Italy, $20 \mathrm{~m}$ ); J: Mojave (California, $12 \mathrm{~m}$ ); K: Kokee (Hawaii, $20 \mathrm{~m}$ ); L: NL-VLBA (Iowa, $25 \mathrm{~m}$ ); M: Miami20 (Florida, $20 \mathrm{~m}$ ); N: NRAO20 (Greenbank, 20 m); O: Ny Ålesund (Norway, 20 m); R: Richmond (Florida, $18 \mathrm{~m}$ ); T: Onsala (Sweden, $20 \mathrm{~m}$ ); V: Wettzell (Germany, $20 \mathrm{~m}$ ); X: Ylow7296 (Yellowknife, $10 \mathrm{~m}$ ); Y: Plattvil (North Carolina, $5 \mathrm{~m}$ ); W: NRAO85 (Green Bank, 26 m). 
standard routines from the AIPS software package, and the images were produced using the self-calibration procedures (e.g., Pearson \& Readhead 1984) of the Caltech DIFMAP software package.

\section{RESULTS}

Throughout this paper we assume a standard Friedmann cosmology with $H_{0}=65 \mathrm{~km} \mathrm{~s}^{-1} \mathrm{Mpc}^{-1}$ and $q_{0}=0.5$. At the distance of OJ $287(z=0.306)$, an angular size of 1 mas corresponds to a linear distance of $4.3 \mathrm{pc}$. The $8 \mathrm{GHz}$ radio maps are presented in Figure 1. The source shows a core-jet structure, with jet components moving in a westerly direction. Table 2 lists the flux, position, and size of each component obtained by model fitting the observed visibilities with elliptical Gaussian components. Position angle is measured from north to east.

The motion of the components away from the core can be better appreciated by inspecting Figure 2, in which the separation of components from the core is plotted against time. The straight lines provide estimates of the presumed zero separation of the components $\mathrm{C} 1$ to $\mathrm{C} 6$ under the assumption of rectilinear motion at constant velocity. The measured proper motions of the components $\mathrm{C} 1$ to $\mathrm{C} 6$ are $0.74 \pm 0.40, \quad 0.44 \pm 0.05, \quad 0.52 \pm 0.09, \quad 0.40 \pm 0.13$, $0.46 \pm 0.05$, and $0.58 \pm 0.07$ mas $^{y^{-1}}{ }^{-1}$, respectively, corresponding to an average superluminal speed of approximately $9 c$. These values are almost twice as high as those seen in VLBI observations made at $5 \mathrm{GHz}$ by Roberts et al. (1987) and Gabuzda et al. (1989). A similar proper motion has also been obtained by Vicente et al. (1996) for the fastest

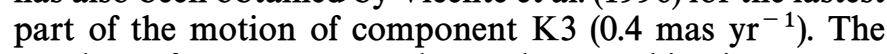
number of components observed over this time range, together with their measured speeds, suggests more frequent ejection of VLBI components than has been previously estimated for this source by Vicente et al. (1996), who concluded that VLBI component ejections occurred at intervals of one-half the orbital period of the putative binary black hole system, or once every 6 yr. The small separation between successive components obtained by Gabuzda \& Cawthorne (1996) at $8 \mathrm{GHz}$ and Tateyama et al. (1996) at 43 $\mathrm{GHz}$ also suggests a higher ejection rate. We can constrain

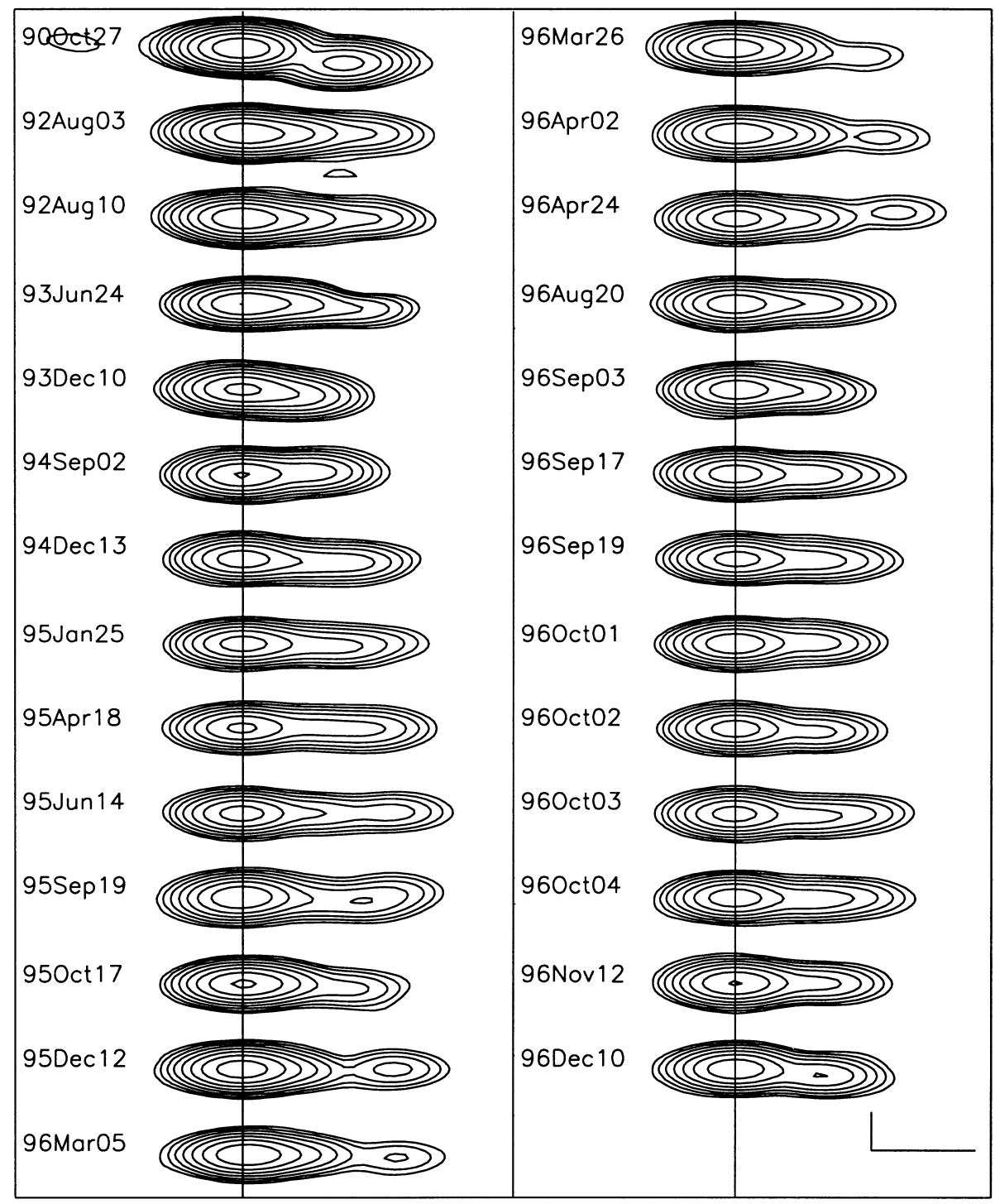

FIG. 1. $-8 \mathrm{GHz}$ VLBI maps of OJ 287. Contour levels increase as $2^{n}$. The lowest contour level is $0.01 \mathrm{Jy} \mathrm{beam}^{-1}$, corresponding to $n=0$. The restored circular beam is 0.6 mas. The stationary component (the VLBI core) is on the east side (left), while the moving components are to the west (right). The tick in the lower part of the map indicates the size in mas. Note that the maps are stretched in right ascension. 
TABLE 2

Model Fits to OJ 287 AT $8 \mathrm{GHz}$

\begin{tabular}{|c|c|c|c|c|c|c|c|}
\hline Epoch & Component & $\begin{array}{l}\text { Flux } \\
\text { (Jy) }\end{array}$ & $\begin{array}{c}r \\
\text { (mas) }\end{array}$ & $\begin{array}{l}\text { P.A. }^{\mathrm{a}} \\
\text { (deg) }\end{array}$ & $\begin{array}{c}\theta^{\mathbf{b}} \\
\text { (mas) }\end{array}$ & $\mathrm{AR}^{\mathrm{c}}$ & $\begin{array}{l}\text { P.A. }^{d} \\
(\mathrm{deg})\end{array}$ \\
\hline \multirow[t]{2}{*}{1990 Oct $27 \ldots \ldots$} & Core & 4.40 & 0.00 & 0 & 0.15 & 0.80 & 0 \\
\hline & $\mathrm{C} 1$ & 1.21 & 0.98 & -109 & 0.70 & 0.80 & 60 \\
\hline \multirow[t]{3}{*}{1992 Aug $03 \ldots \ldots$} & Core & 2.20 & 0.00 & 0 & 0.19 & 0.80 & 80 \\
\hline & $\mathrm{C} 3$ & 0.76 & 0.51 & -98 & 0.30 & 0.80 & -38 \\
\hline & $\mathrm{C} 2$ & 0.21 & 1.24 & -110 & 1.10 & 0.80 & 5 \\
\hline \multirow{3}{*}{1992 Aug $10 \ldots \ldots$} & Core & 2.30 & 0.00 & 0 & 0.17 & 0.80 & -60 \\
\hline & $\mathrm{C} 3$ & 0.55 & 0.49 & -99 & 0.30 & 0.80 & -45 \\
\hline & $\mathrm{C} 2$ & 0.27 & 1.17 & -110 & 0.90 & 0.70 & 50 \\
\hline \multirow[t]{3}{*}{1993 Jun $24 \ldots \ldots$} & Core & 1.05 & 0.00 & 0 & 0.15 & 0.70 & 0 \\
\hline & $\mathrm{C} 4$ & 0.61 & 0.39 & -89 & 0.54 & 0.60 & 105 \\
\hline & $\mathrm{C} 3$ & 0.23 & 0.98 & -99 & 1.10 & 0.70 & -70 \\
\hline \multirow[t]{3}{*}{1993 Dec $10 \ldots \ldots$} & Core & 1.56 & 0.00 & 0 & 0.19 & 1.00 & \\
\hline & $\mathrm{C} 4$ & 0.54 & 0.55 & -102 & 0.30 & 0.70 & -10 \\
\hline & $\mathrm{C} 3$ & 0.48 & 1.19 & -85 & 0.80 & 0.50 & 0 \\
\hline \multirow[t]{2}{*}{1994 Sep $02 \ldots \ldots$} & Core & 1.25 & 0.00 & 0 & 0.10 & 1.00 & \\
\hline & $\mathrm{C} 5$ & 0.55 & 0.62 & -88 & 0.40 & 1.00 & \\
\hline \multirow[t]{2}{*}{$1994 \operatorname{Dec} 13 \ldots \ldots$} & Core & 0.93 & 0.00 & 0 & 0.13 & 0.80 & -10 \\
\hline & $\mathrm{C} 5$ & 0.89 & 0.79 & -95 & 0.80 & 0.80 & 90 \\
\hline \multirow[t]{2}{*}{1995 Jan $25 \ldots \ldots$} & Core & 0.97 & 0.00 & 0 & 0.17 & 0.90 & -75 \\
\hline & $\mathrm{C} 5$ & 0.54 & 0.82 & -93 & 0.70 & 0.80 & -90 \\
\hline \multirow{2}{*}{1995 Apr 18 ...... } & Core & 0.76 & 0.00 & 0 & 0.17 & 0.80 & -80 \\
\hline & $\mathrm{C} 5$ & 0.58 & 0.98 & -90 & 0.90 & 0.80 & 80 \\
\hline \multirow[t]{2}{*}{1995 Jun $14 \ldots \ldots$} & Core & 0.83 & 0.00 & 0 & 0.17 & 0.70 & -41 \\
\hline & $\mathrm{C} 5$ & 0.28 & 1.06 & -99 & 1.16 & 0.70 & 10 \\
\hline \multirow[t]{2}{*}{1995 Sep 19 ..... } & Core & 1.26 & 0.00 & 0 & 0.20 & 0.80 & -50 \\
\hline & $\mathrm{C} 5$ & 0.40 & 1.13 & -96 & 0.93 & 0.78 & -8 \\
\hline \multirow[t]{3}{*}{1995 Oct $17 \ldots \ldots$} & Core & 1.24 & 0.00 & 0 & 0.12 & 0.70 & 50 \\
\hline & $\mathrm{C} 6$ & 0.36 & 0.30 & -86 & 0.35 & 1.00 & \\
\hline & $\mathrm{C} 5$ & 0.18 & 1.13 & -98 & 1.08 & 1.00 & \\
\hline \multirow[t]{3}{*}{1995 Dec $12 \ldots \ldots$} & Core & 1.92 & 0.00 & 0 & 0.11 & 0.80 & -50 \\
\hline & $\mathrm{C} 6$ & 0.24 & 0.36 & -97 & 0.25 & 0.83 & -56 \\
\hline & $\mathrm{C} 5$ & 0.28 & 1.30 & -90 & 1.30 & 0.90 & -86 \\
\hline 1996 Mar $05 . . . .$. & Core & 1.18 & 0.00 & 0 & 0.17 & 0.80 & -73 \\
\hline & $\mathrm{C} 6$ & 0.24 & 0.43 & -89 & 0.35 & 0.90 & -10 \\
\hline & $\mathrm{C} 5$ & 0.12 & 1.35 & -99 & 1.13 & 0.90 & 60 \\
\hline 1996 Mar $26 \ldots \ldots$ & Core & 0.95 & 0.00 & 0 & 0.17 & 0.70 & -70 \\
\hline & $\mathrm{C} 6$ & 0.35 & 0.38 & -95 & 0.38 & 0.90 & -1 \\
\hline & $\mathrm{C} 5$ & 0.13 & 1.33 & -108 & 1.20 & 0.75 & 3 \\
\hline 1996 Apr $02 \ldots \ldots$ & Core & 1.12 & 0.00 & 0 & 0.17 & 0.80 & -75 \\
\hline & $\mathrm{C} 6$ & 0.30 & 0.42 & -90 & 0.25 & 0.70 & 50 \\
\hline & $\mathrm{C} 5$ & 0.03 & 1.39 & -100 & 1.12 & 0.60 & 10 \\
\hline 1996 Apr 24 . & Core & 0.92 & 0.00 & 0 & 0.15 & 0.80 & 0 \\
\hline & $\mathrm{C} 6$ & 0.24 & 0.59 & -90 & 0.43 & 0.80 & -80 \\
\hline & $\mathrm{C} 5$ & 0.05 & 1.42 & -99 & 1.49 & 0.80 & -63 \\
\hline 1996 Aug $20 \ldots \ldots$ & Core & 1.06 & 0.00 & 0 & 0.12 & 0.70 & 80 \\
\hline & $\mathrm{C} 6$ & 0.45 & 0.70 & -89 & 0.50 & 0.80 & 85 \\
\hline 1996 Sep $03 \ldots \ldots$ & Core & 0.93 & 0.00 & 0 & 0.12 & 0.80 & 81 \\
\hline & $\mathrm{C} 6$ & 0.42 & 0.73 & -95 & 0.42 & 0.70 & -66 \\
\hline 1996 Sep $17 \ldots \ldots$ & Core & 1.03 & 0.00 & 0 & 0.15 & 0.70 & 62 \\
\hline & C6 & 0.35 & 0.78 & -92 & 0.43 & 0.80 & 84 \\
\hline 1996 Sep 19 ..... & Core & 0.91 & 0.00 & 0 & 0.17 & 0.70 & 3 \\
\hline & $\mathrm{C} 6$ & 0.33 & 0.80 & -92 & 0.40 & 0.80 & 80 \\
\hline 1996 Oct $01 \ldots \ldots$ & Core & 1.24 & 0.00 & 0 & 0.18 & 0.80 & 18 \\
\hline & $\mathrm{C} 6$ & 0.32 & 0.79 & -86 & 0.40 & 0.71 & 56 \\
\hline 1996 Oct $02 \ldots \ldots$ & Core & 0.99 & 0.00 & 0 & 0.17 & 0.80 & -12 \\
\hline & $\mathrm{C} 6$ & 0.27 & 0.82 & -96 & 0.48 & 0.61 & -59 \\
\hline 1996 Oct $03 \ldots \ldots$ & Core & 0.89 & 0.00 & 0 & 0.16 & 0.80 & -18 \\
\hline & $\mathrm{C} 6$ & 0.43 & 0.84 & -97 & 0.85 & 0.70 & -80 \\
\hline 1996 Oct $04 \ldots \ldots$ & Core & 1.03 & 0.00 & 0 & 0.16 & 0.80 & -6 \\
\hline & $\mathrm{C} 6$ & 0.37 & 0.84 & -92 & 0.50 & 0.80 & -85 \\
\hline 1996 Nov $12 \ldots \ldots$ & Core & 1.41 & 0.00 & 0 & 0.17 & 0.80 & 20 \\
\hline & $\mathrm{C} 6$ & 0.43 & 0.87 & -91 & 0.66 & 0.60 & 84 \\
\hline 1996 Dec $10 \ldots \ldots$ & Core & 1.16 & 0.00 & 0 & 0.18 & 0.80 & -135 \\
\hline & C6 & 0.49 & 0.86 & -98 & 0.50 & 0.60 & 70 \\
\hline
\end{tabular}

${ }^{\text {a }}$ Position angle of the component.

${ }^{b}$ Major axis of the elliptical component.

${ }^{c}$ Axial ratio of the elliptical component.

${ }^{d}$ Position angle of the major axis of the elliptical component. 


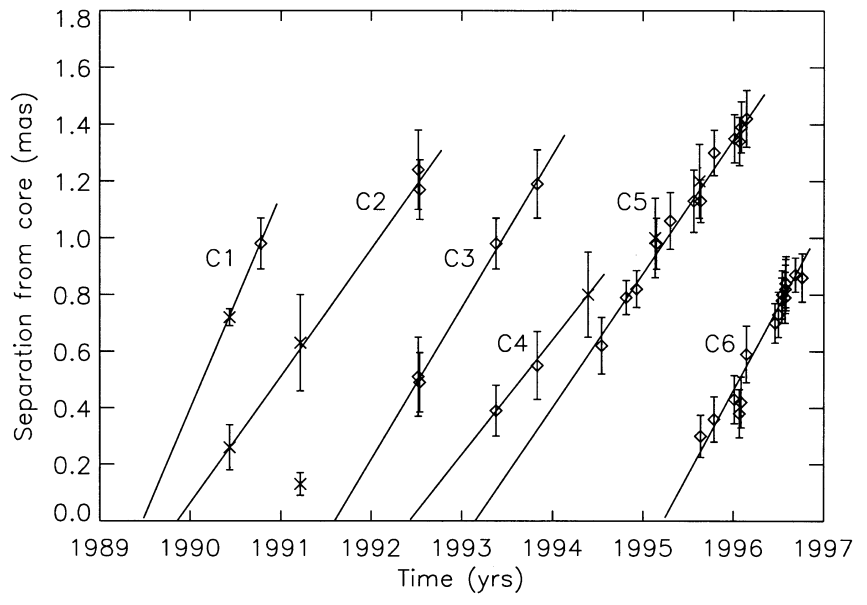

FIG. 2.-Motion of components in OJ 287. The vertical axis shows the separation in mas of the center of the component from the core. The straight lines provide estimates of the presumed zero separation of components $\mathrm{C} 1$ to $\mathrm{C} 6$ under the assumption of rectilinear motion at constant velocity. Diamonds represent our data and crosses published data. At 1990.47 we show $8 \mathrm{GHz}$ data from Gabuzda \& Cawthorne (1996), at 1991.27 $43 \mathrm{GHz}$ data from Tateyama et al. (1996), at $1994.528 \mathrm{GHz}$ data from Fey et al. (1996), and at 1995.28 and $1995.778 \mathrm{GHz}$ data from Fey \& Charlot (1997). The error bars on component positions are assumed to be one-eighth of the beam width.

these results using radio light curves, provided that radio outbursts are connected with VLBI components.

\section{DISCUSSION}

\subsection{Radio Light Curves}

Figure 3 shows the optical and radio light curves of OJ 287. The beginning of each radio outburst is indicated by a vertical line. There is a good agreement between the epochs of the radio outbursts and the epochs of zero separation of

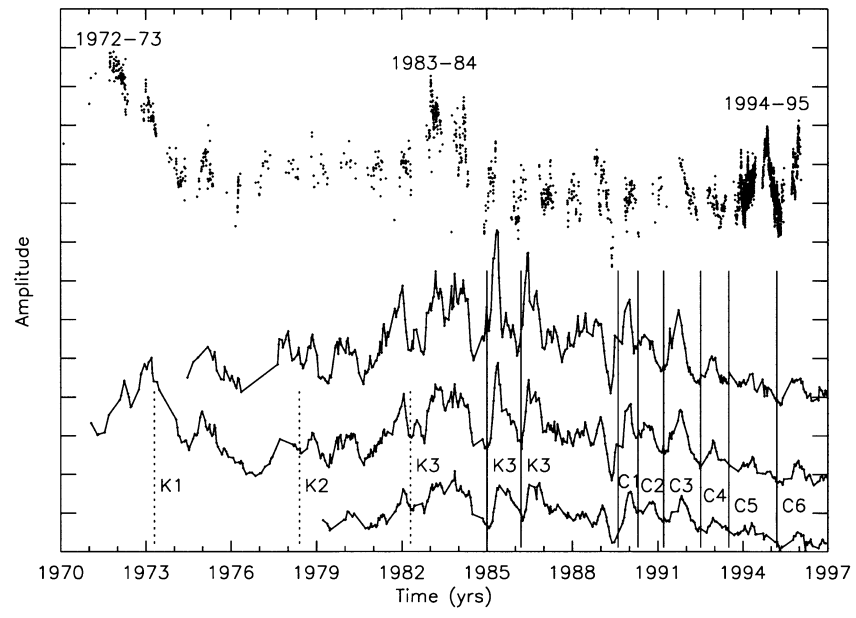

FIG. 3.-Optical and radio light curves of OJ 287. The optical data are from Fig. 2 of Sillanpää et al. (1996). The radio data are from the University of Michigan Radio Astronomy Observatory. From top to bottom the plot shows $V$-band optical data, and 15,8 , and $5 \mathrm{GHz}$ radio data. Each tick mark on the ordinate corresponds to a flux density of $2 \mathrm{Jy}$ for the radio data, and an amplitude of 1 mag for the optical data. The vertical lines indicate the beginning of the outburst related to each VLBI component. The dashed lines indicate the epochs of the birth of components $\mathrm{K} 1, \mathrm{~K} 2$, and K3. The multiple lines shown for component K3 are discussed in the text. The epochs of double-peaked optical outbursts are indicated over each such outburst. components C1 to C6, which are 1989.5, 1990.0, 1991.7, 1992.5, 1993.3, and 1995.4, respectively. Only C2 would require a higher proper motion for its zero separation epoch to match the beginning of the radio outburst around 1990.2. However, we can place the $43 \mathrm{GHz}$ data point a little closer to the core, accounting for a possible frequency-dependent separation between it and the $8 \mathrm{GHz}$ data. Since the $8 \mathrm{GHz}$ measurement of Gabuzda \& Cawthorne (1996) is not clearly resolved from the core, it can also be placed closer to the core, resulting in a zero-separation epoch for $\mathrm{C} 2$ more consistent with the beginning of the outburst.

The zero-separation epoch of $\mathrm{C} 1$ is estimated from only two points. The point closer to the core was taken from 8 $\mathrm{GHz}$ VLBI observations obtained by Gabuzda \& Cawthorne (1996). The extrapolation of the fitted motion to zero separation agrees well with the start of the radio outburst at 1989.5. Component C3 would be related to the outburst that started in 1991.5. There is also a weak knot observed near the core in 1991.27 at $43 \mathrm{GHz}$, which may be either component $\mathrm{C} 3$ itself or another component. The radio light curve shows a substructure that may be linked with this component, suggesting that it might have been an intrinsically weak or short-lived component that faded or merged with $\mathrm{C}$ 3. Component $\mathrm{C} 4$ has three data points; the farthest point from the core is a measurement obtained from VLBA data (Fey, Clegg, \& Fomalont 1996). This component is associated with a single radio outburst that started in 1992.5. The next component, C5, has the largest separation from the core, and is probably associated with a radio outburst that started in 1993.4. Two points from VLBA data at $8 \mathrm{GHz}$ (Fey \& Charlot 1997) have been included in the curve. The small separation between $\mathrm{C} 4$ and $\mathrm{C} 5$ raises the question of whether they can be regarded as one component. In the one radio outburst per VLBI component scheme, $\mathrm{C} 4$ and $\mathrm{C} 5$ are individual components that merge later into $\mathrm{C} 5$. One weak point in this interpretation is the nondetection of C5 closer to the core in the 1993 December data. However, it could be too close to $\mathrm{C} 4$ to be discernible as a separate component at this time. If $\mathrm{C} 4$ and $\mathrm{C} 5$ are separate components, we would expect a merging of these two components around the end of 1994. In fact, an increase in the flux density of component C5 in 1994 December could be taken as an indication of such an event. Finally, component $\mathrm{C} 6$ could be related to the outburst that started in 1995.4.

We have also examined a series of geodetic VLBI maps at $8 \mathrm{GHz}$ obtained by Vicente et al. (1996). They have interpreted the structure of OJ 287 in terms of helical motion, with an indication of a small loop at a distance of about 0.6 mas from the core. They claim that a second loop at 2.4 mas is consistent with VLBI data at $5 \mathrm{GHz}$ (Roberts et al. 1987; Gabuzda et al. 1989). Even so, they found no evidence of a boosting of $\mathrm{K} 3$, as would be expected from the geometrical effects of a helically moving feature, and the positions of $\mathrm{K} 2$ appeared to be well below the calculated helical trajectory. Even revising the K2 positions upward (Vicente et al. 1996), the fit does not improve much. Now, if we are allowed to break the component corresponding to epoch 1986.81 (Fig. 3 of Vicente et al. 1996) into two subcomponents at 0.4 and 0.8 mas, a resolution of 0.5 mas would hardly distinguish between the two models. We could then have rectilinear motion for both components, with a proper motion of about 0.4 mas $\mathrm{yr}^{-1}$. An appealing support for the presence of two rectilinear components is the existence of well- 
defined radio outbursts corresponding to their estimated zero-separation epochs, as shown on Figure 3.

We have also looked for VLBI/radio outburst correlations for the older VLBI components $\mathrm{K} 1, \mathrm{~K} 2$, and $\mathrm{K} 3$ (Roberts et al. 1987). The derived proper motion for these components $\left(0.20-0.28\right.$ mas $\left.^{-1}\right)$ is about twice as low as our measured proper motion. This indicates that in the past, different components may have been regarded as the same component. The imbalance between the number of knots and the number of radio outbursts is also noticeable in Figure 3. There are at least two well-defined radio outbursts between the ones related to the births of K2 in 1978.4 and K3 in 1982.3 (Roberts et al. 1987). A similar effect is also present for component $\mathrm{K} 1$, indicating that VLBI components may have been missed.

\subsection{Optical Light Curve}

The top part of Figure 3 shows the optical light curve from Sillanpää et al. (1996). There is a remarkable similarity between it and the radio light curves. Despite the complexity of the structures, most features can be found in both emission bands. The most easily recognizable correlated radio-optical feature is the double structure that occurred in 1983-1984, which had a similar amplitude in the radio and the optical. The double outbursts of 1971-1973 and 19941995 present a pair of almost identical optical flares, while in the radio these features appear almost as single outbursts coincident with the second optical flare. However, upon more detailed inspection, small protrusions on the radio light curves coincident with the first flare of the double peak are also present in 1971 and 1994. Even weak optical-radio substructures can be pinpointed in the feature beginning in 1994. This can be seen in Figure 4, which shows an expanded section of the light curve corresponding to the period of VLBI observations (1990-1997). It is also clear that radio features are delayed by a few months relative to optical features. The inclined dashed lines on the figure correspond to a delay of $0.14 \mathrm{yr}$. The smoother profile at lower frequencies and the time delay between the optical and radio

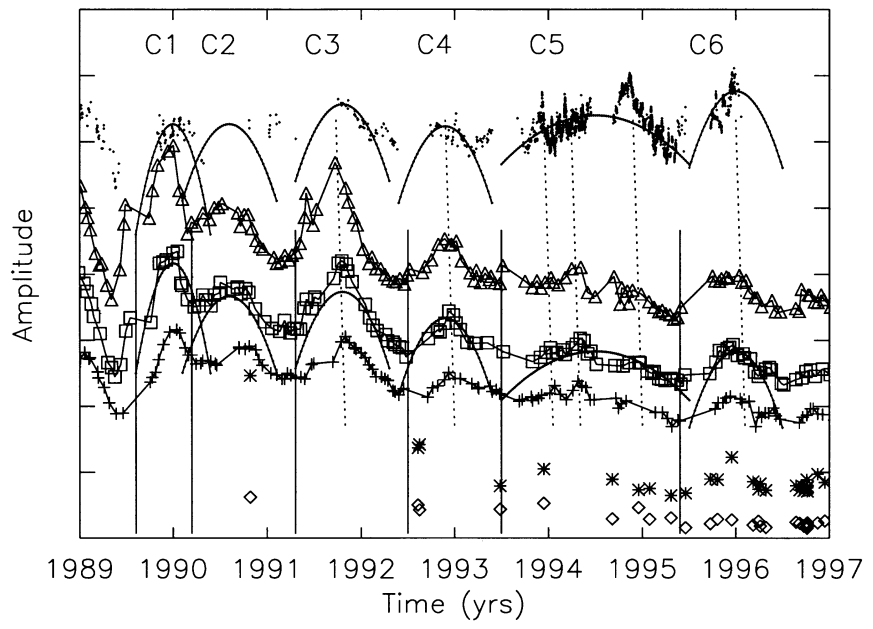

FIG. 4.-Optical, radio, and VLBI light curves. The "arcs" represent the size and shape of the outburst associated with each VLBI component. They are plotted on the optical and $8 \mathrm{GHz}$ data. Below the radio light curves the flux of the core (asterisks) and the sum of the flux of the jet components (diamonds) are also shown. Except for the optical data, the relative amplitudes are all on the same scale. Each tick mark on the ordinate corresponds to a flux density of $2 \mathrm{Jy}$ for the radio data, and an amplitude of 2 mag for the optical data. bands gives strong support for the synchrotron selfabsorption process (Melnikov \& Magun 1998). We propose that radio-optical structures are self-absorbed synchrotron sources, and the absence of radio features not correlated with optical features may be due to a more compact synchrotron source.

\subsection{VLBI Light Curves}

Provided that radio and optical emission are synchrotron, it remains to be seen whether this emission originates in the core, the jet, or the accretion disk. The high-density time coverage offered by the geodetic VLBI observations, particularly for components $\mathrm{C} 5$ and C6, enabled us to study the evolution of the flux of the VLBI components (core and moving components) with time, as shown in Figure 4 along with the radio light curves. It is clear that the time variation of the flux of the core closely follows the shape of the radio light curves, while the flux of the moving components remains nearly constant with time. With less data, similar results can be seen for C4 in 1993 December. The same behavior can also be seen in geodetic VLBI data studied by Vicente et al. (1996), as shown in Figure 5. Another aspect of the flux of the moving components is that it does not depend on the strength of the radio outbursts. For instance, a radio outburst peaking at $7 \mathrm{Jy}$ (K3 of Vicente et al. 1996) has an associated moving component with a flux similar to those associated with outbursts peaking at $2 \mathrm{Jy} \mathrm{(e.g.,} \mathrm{C6} \mathrm{of}$ present work). These results seem to indicate that the VLBI components contribute to the profile of the radio light curve only when they are just emerging from the core at the time of a radio outburst, and that they are still merged with the core at the resolution of these VLBI observations.

\subsection{Binary Systems}

We may speculate that the flares are related to an increase in the accretion rate being induced by the passage of the companion mass (Sillanpää et al. 1988). We assume that the synchrotron emission is optically thin at optical bands and self-absorbed at radio bands. The absence of radio outbursts coincident with the first peak of the double-

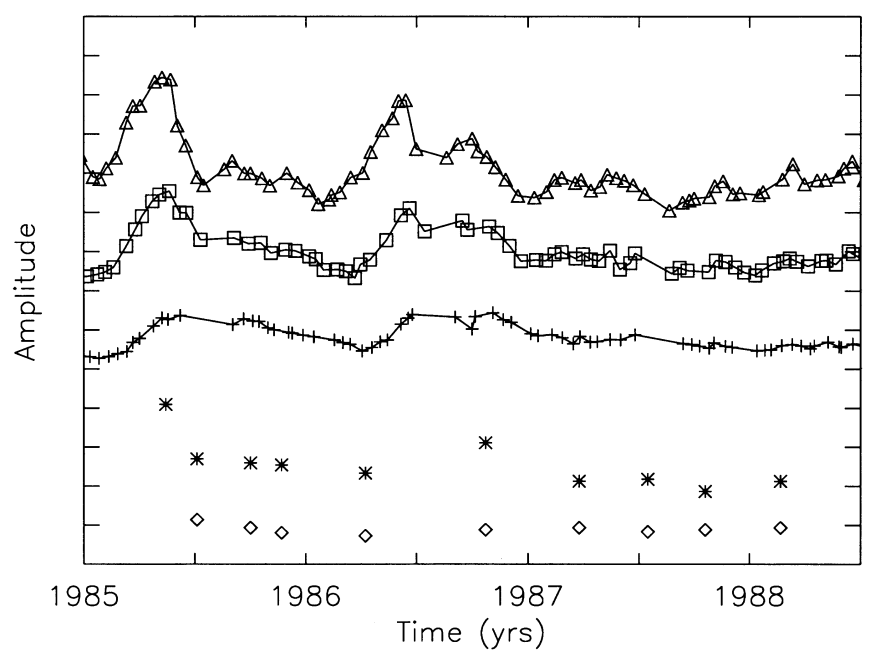

FIG. 5.-Radio light curves compared to VLBI light curves from Vicente et al. (1996). Below the radio light curves are shown the flux of the core (asterisks) and the sum of the flux of the jet components (diamonds) from Vicente et al. (1996). All amplitudes are on the same scale. Each tick mark on the ordinate corresponds to a flux density of $2 \mathrm{Jy}$. 
peaked optical outbursts could be an indication of a more compact synchrotron source. We also assume that the major flux variations come from the core. Keeping in mind a synchrotron emission mechanism for optical and radio outbursts and knowing that radio outbursts have irregular periods of about a year while major optical flares appear every $12 \mathrm{yr}$, we can conceive a binary model in which the roughly annual outbursts of the core-jet system of the primary are visited every $12 \mathrm{yr}$ by the secondary, increasing the flux of the outbursts.

A narrow jet placed on the disk axis of either the coplanar binary model of Sillanpää et al. (1988) or the steep inclined orbit model of Lehto \& Valtonen (1996) would provide an appropriate scenario to explain the observed evolution of the VLBI components as well as the radio and optical outbursts. Lehto \& Valtonen (1996) have already suggested a narrow cone on the disk axis to explain a sudden fading "eclipse" caused by the secondary passing over the line of sight. However, those binary systems based on the sweeping beam models of Villata et al. (1996) and Katz (1997) would be discarded, because they would not account for the observed evolution of the VLBI components and the number of radio outbursts. While a binary system through its accretion disk increases the accretion rate at evenly spaced intervals, the nonperiodic nature of nonthermal outbursts would spread the exact timing of the observable effects of this increased accretion rate. This is indeed in accordance with the small variation observed around the period of $12 \mathrm{yr}$. It would also be possible, depending on the duration of the increased accretion rate and the timing of the irregular ejection rate, to observe a flare with a triple structure, as reported by Sundelius et al. (1997).

\section{CONCLUSIONS}

We have presented 27 geodetic VLBI maps obtained from the Washington VLBI Correlator Facility at the US
Naval Observatory. These maps show a sequence of six VLBI components associated with radio outbursts. The proper motion of the components was found to be around $0.5 \mathrm{mas} \mathrm{yr}^{-1}$, almost twice as high as that seen in previous VLBI observations of this source. Such a higher proper motion, along with the larger number of VLBI components, implies a higher component ejection rate for OJ 287, a result supported by the close relationship between the radio outbursts and the appearance of VLBI components.

We have assumed that the radio-optical outbursts are synchrotron emission. The increase in the accretion rate due to the pericenter passage of the companion mass would not directly produce a VLBI component or radio outburst, but would rather provide a means to energize the system and increase the flux of the synchrotron emission. The irregular appearance of radio outbursts/VLBI components (about once a year), which is intrinsic to the engine, operates continuously. Every $12 \mathrm{yr}$ the system is more apt to produce a higher flux; however, the ejection rate of VLBI components and hence the number of radio outbursts is not affected by this process.

C. E. T. thanks the grant received from the Fundação de Amparo a Pesquisa do Estado de São Paulo (FAPESP; proc. 96/6267-1) to undertake 3 months of work with geodetic VLBI data at the US Naval Observatory (USNO). We also thank Aimo Sillanpää for supplying the optical data and the referee for his suggestions and comments, which helped with the improvement of the paper. The University of Michigan Radio Astronomy Observatory is supported by the National Science Foundation and by funds from the University of Michigan. The Fortaleza VLBI facility was built and is operated with partial support from NASA, USNO, and NOAA, the Brazil ministry of Science and Technology, MCT-FINEP, Mackenzie, INPE, and CRAAE (joint center of Mackenzie, INPE, USP, and UNICAMP, Brazil).

\section{REFERENCES}

Britzen, S., Witzel, A., Gontier, A. M., Schalinski, C. J., \& Campbell, J. 1994, in IAU Symp. 159, Multi-Wavelength Continuum Emission of AGN, ed. T. J. L. Courvosier \& A. Blecha (Dordrecht: Kulwer), 423

Carter, W. E., Robertson, D. S., \& MacKay, J. R. 1985, J. Geophys. Res., 90,4577

Charlot, P. 1990, A\&A, 229, 51

Coates, R. J., Frey, H., Mead, G. D., \& Bosworth, J. M. 1985, IEEE Trans. Geosci. Remote Sensing, GE-23, 360

Eubanks, T. M., et al. 1991, in IERS Technical Note No. 8 (Paris: IERS), 73

Fey, A. F., \& Charlot, P. 1997, ApJS, 111, 95

Fey, A. F., Clegg, A. W., \& Fomalont, E. B. 1996, ApJS, 105, 299

Gabuzda, D. C., \& Cawthorne, T. V. 1996, MNRAS, 283, 759

Gabuzda, D. C., Wardle, J. F. C., \& Roberts, D. H. 1989, ApJ, 336, L59

Guoquiang, T., Rönnäng, B., \& Bååth, L. 1987, A\&A, 185, 87

Hutchings, J. B., Crampton, D., Campbell, B., Duncan, D., \& Glendenning, B. 1984, ApJS, 55, 319

Katz, J. I. 1997, ApJ, 478, 527

Kinman, T. D. 1975, in IAU Symp. 67, Variable Star and Stellar Evolution, ed V. E. Sherwood \& L. Plaut (Dordrecht: Reidel), 573

Lehto, H. J., \& Valtonen, M. J. 1996, ApJ, 460, 207

Melnikov, V., \& Magun, A. 1998, in CESRA Workshop on Coronal Explosive Events (Finland, 1998 June 9-13) (Helsinki: Metsahovi Publications on Radio Science), 54
Miller, J. S., French, H. B., \& Hawley, S. A. 1978, in Pittsburgh Conference on BL Lac Objects, ed. A. M. Wolfe (Pittsburgh: Univ. Pittsburgh), 176 Pearson, T. J., \& Readhead, A. C. S. 1984, ARA\&A, 22, 97

Piner, B. G., \& Kingham, K. A. 1998, ApJ, 507, 706

Roberts, D. H., Gabuzda, D. C., \& Wardle, J. F. C. 1987, ApJ, 323, 536

Rogers, A. E. E., et al. 1983, Science, 219, 51

Sillanpää, A., Haarala, S., Valtonen, M. J., Sundelius, B., \& Byrd, G. G. $1988, \mathrm{ApJ}, 325,628$

Sillanpää, A., et al. 1996, A\&A, 315, L13

Sitko, M. L., \& Junkkarinen, V. T. 1985, PASP, 97, 1158

Smith, D. E., \& Turcotte, D. L., ed. 1993, Contributions of Space Geodesy to Geodynamics: Crustal Dynamics (Washington: American Geophys. Union)

Sundelius, B., Wahde, M., Lehto, H. J., \& Valtonen, M. J. 1997, ApJ, 484, 180

Tateyama, C. E., et al. 1996, PASJ, 48, 37

Tateyama, C. E., Kingham, K. A., Kaufmann, P., Piner, B. G., de Lucena, A. M. P., \& Botti, L. C. L. 1998, ApJ, 500, 810

Usher, P. D. 1979, AJ, 84, 1253

Valtaoja, L., Sillanpää, A., \& Valtaoja, I. 1987, A\&A, 184, 57

Vicente, L., Charlot, P., \& Sol, H. 1996, A\&A, 312, 727

Villata, M., Raiteri, C. M., Silanpää, A., \& Takalo, L. O. 1998, MNRAS, 293, L13 